\title{
Exploring Barriers to Medication Adherence Using COM-B Model of Behaviour Among Patients with Cardiovascular Diseases in Low- and Middle-Income Countries: A Qualitative Study
}

\author{
Pallavi Mishra' \\ Ajay S Vamadevan ${ }^{1,2}$ \\ Ambuj Roy ${ }^{3}$ \\ Rohit Bhatia ${ }^{4}$ \\ Nitish Naik ${ }^{3}$ \\ Sandeep Singh ${ }^{3}$ \\ Gideon Senyo Amevinya ${ }^{5}$ \\ Ernest Amoah Ampah ${ }^{5}$ \\ Yolanda Fernandez ${ }^{6}$ \\ Caroline Free ${ }^{7}$ \\ Amos Laar ${ }^{5}$ \\ Dorairaj Prabhakaran ${ }^{1,8,9}$ \\ Pablo Perel $\mathbb{D D}^{6}$ \\ Helena Legido-Quigley ${ }^{10,11}$ \\ 'Health Systems Unit, Centre for Chronic \\ Disease Control, New Delhi, India; ${ }^{2} \mathrm{Goa}$ \\ Institute of Management, Goa, India; \\ ${ }^{3}$ Department of Cardiology, All India Institute \\ of Medical Science, New Delhi, India; \\ ${ }^{4}$ Department of Neurology, All India Institute \\ of Medical Science, New Delhi, India; \\ ${ }^{5}$ Department of Population, Family \& \\ Reproductive Health, School of Public Health, \\ University of Ghana, Accra, Ghana; ${ }^{6}$ Centre for \\ Global Chronic Conditions, London School of \\ Hygiene and Tropical Medicine, London, UK; \\ ${ }^{7}$ Department of Population Health, London \\ School of Hygiene and Tropical Medicine, \\ London, UK; ${ }^{8}$ Department of Non- \\ Communicable Disease Epidemiology, London \\ School of Hygiene and Tropical Medicine, \\ London, UK; ${ }^{9}$ Research and Policy, Public \\ Health Foundation of India, Gurugram, India; \\ ${ }^{10}$ Saw Swee Hock School of Public Health, \\ National University of Singapore, Singapore; \\ "Department of Health Services Research \& \\ Policy, London School of Hygiene and Tropical \\ Medicine, London, UK
}

Correspondence: Pablo Perel

Centre for Global Chronic Conditions,

London School of Hygiene \& Tropical

Medicine (LSHTM), Keppel Street,

London, WCIE 7HT, UK

Tel +442076368636

Email Pablo.Perel@Ishtm.ac.uk
Introduction: In 2016, cardiovascular diseases (CVDs) led to 17.9 million deaths worldwide, representing $31 \%$ of all global deaths. CVDs are the leading cause of mortality worldwide and significant barriers to achieving the sustainable development goals. Modern medicines have been significant in improving health outcomes. However, non-adherence to medication is one of the reasons behind adverse health-related outcomes among patients suffering from atherosclerotic cardiovascular disease in low- and middle-income countries.

Patients and Methods: This qualitative study was conducted at two tertiary care hospitals in India and Ghana. A total of 35 in-depth interviews were conducted with atherosclerosis cardiovascular disease (ASCVD) patients. The data were analysed thematically using the Capability Opportunity and Motivation (COM-B) framework.

Findings: The findings were summarised under three important broad themes of the COM-B framework: capability, opportunity and behaviour. Under capability, comprehension of disease, medication schedule, and unplanned travel affected adherence among patients. Cost of medication, insurance and access were the critical factors under opportunity, which negatively influenced medication adherence. Mood, beliefs about treatment and outcome expectations under motivation led to non-adherence among patients. Apart from these factors, some important health system factors such as health care experience and trust in the facilities and reliance on alternative medication also affected adherence in both countries.

Conclusion: This study has highlighted that the health system factors have dominantly influenced adherence to medication in India and Ghana. In India, we found participants to be satisfied with their health care provided at the government hospitals. However, limited time for consultation, lack of well-stocked pharmacy and unclear prescription negatively influenced adherence among participants in India and Ghana. The study emphasises that the health system needs to be strengthened, and the patients' belief system needs to be explored to address the issue of medication adherence in LMICs.

Keywords: non-communicable diseases, atherosclerotic cardiovascular disease, public health, qualitative research

\section{Introduction}

In 2016, Cardiovascular Diseases (CVDs) led to 17.9 million deaths worldwide, which represented $31 \%$ of all global deaths. ${ }^{1}$ The analysis of the Global Burden of Disease study 2016, reveals that CVDs represents $24 \%$ of the Non-communicable Diseases (NCDs) related DALYs. ${ }^{2}$ Studies show that in 2015 alone, low and 
middle-income countries (LMICs) accounted for more than five million deaths due to CVDs (WHO, 2017). LMICs bear the largest burden of CVDs, and most have limited resources to curtail the increasing burden of CVDs. $^{3}$

Various population-based studies have found an association between socio-demographic characteristics such as increasing age, gender and lower education, and the increasing burden of CVDs. ${ }^{4}$ Other factors contributing to the rise in CVDs are metabolic (high blood pressure, high blood glucose, dyslipidemias, obesity) and behavioural risk factors (tobacco use, unhealthy diet, physical inactivity). ${ }^{5}$

Modern medicines have played a significant role in improving health outcomes. However, non-adherence to medication is one of the reasons behind adverse healthrelated outcomes among atherosclerotic cardiovascular disease (ASCVD) patients. ${ }^{6,7}$ Adherence to the medication has been defined as "active, voluntary, and collaborative involvement of the patient in a mutually acceptable course of behaviour to produce a therapeutic result". 8 Therefore, non-adherence to medication is a behaviour choice which is determined by psychosocial, economic, and health system factors.

One of the effective ways of averting CVD mortality is secondary prevention, which includes strategies that effectively reduce the probability of recurrence of a cardiovascular event in patients with known atherosclerotic CVDs. ${ }^{9}$ Risk factor modification, counselling, and medication adherence can help reduce CVD-related morbidity and mortality. ${ }^{10}$

The study took place in Ghana and India. According to WHO estimates, in Ghana, the probability of dying from NCDs, including CVDs, within the age group of 30 to 70 years is $20.8 \% .^{11}$ Many Ghanaians with NCDs such as high blood pressure are unaware of their condition, and of those who are aware and on treatment, less than 5\% have controlled blood pressure. ${ }^{12,13}$ The referral facility tends to receive these cases at a late stage of the illness, and this further burdens the health system of the country. Similarly, CVDs are the leading cause of mortality in India. ${ }^{14}$ As the largest proportion of the population, lower socio-economic groups contribute most to the burden of CVDs risk factors in India. ${ }^{15}$ A study hypothesized that a combination of four drugs (aspirin, a $\beta$-blocker, a statin, and an angiotensinconverting enzyme [ACE] inhibitor) could reduce CVD events by $75 \%$ in those with vascular disease. ${ }^{16}$ However, the PURE study registered low usage of the combination of these four drugs in LMICs. ${ }^{17}$

The focus of this paper is to understand existing local barriers to medication use, including socio-psychological, economic, and health system barriers in two LMICs-India and Ghana. This paper is part of a larger study (Txt2Heart) which aimed first to understand the barriers for using medication for ASCVD and then, using behavioural change techniques, develop SMS messages to improve medication adherence in these settings.

\section{Methods}

\section{The Study Setting}

In Ghana, participants recruited for this study were patients diagnosed with CVDs receiving treatment from a tertiary health facility. The hospital is situated in the La Dade Kotopon District of Greater Accra Region. The district is much urbanised and serves as both a domicile and commercial setting for its population. The Hospital is a referral health facility within the country and provides general healthcare services for patients in and out of the national capital, Accra. It offers services for patients on the country's National Health Insurance Scheme (NHIS) as well as for private health insurance and out of pocket patients.

In India, participants were recruited from a tertiary care hospital in New Delhi, India. The hospital provides health care to CVD and other patients from all across India.

\section{Sampling}

ASCVD patients from the tertiary care Hospital in Ghana were identified retrospectively through a desk review of patients' folders, and prospectively in India by one on one interaction with patients visiting the outpatient department (OPD) at the hospital, in New Delhi, on their scheduled check-up.

In-depth interviews (IDIs) and focus group discussions (FGDs) were conducted with 35 (16 from India and 19 from Ghana) purposively selected participants using a topic guide. Participants were included if they were over 18 years old with six months of history of established ASCVD (See Table 1).

Trained research staff from the Centre for Chronic Disease Control (CCDC), India, and the School of Public Health, University of Ghana collected the data. The IDIs and FGDs were conducted in the local language. Hindi 
Table I Participant Characteristics

\begin{tabular}{|l|l|l|}
\hline & India & Ghana \\
\hline IDIs & 16 & 19 \\
\hline FGDs & 1 & 3 \\
\hline Male participant & 10 & 9 \\
\hline Female participant & 06 & 10 \\
\hline Mean age & 51.37 & 61.74 \\
\hline known Ischaemic Heart Disease (IHD) & 8 & 2 \\
\hline Ischemic stroke & 4 & 13 \\
\hline Myocardial infarction & 3 & 1 \\
\hline Coronary Artery Bypass Graft (CABG) & 1 & 0 \\
\hline Cardiomyopathy & 0 & 1 \\
\hline Acute Coronary Syndrome (ACS) & 0 & 1 \\
\hline Multivalvular Heart Disease & 0 & I \\
\hline
\end{tabular}

was the preferred language of the participants in India, and in Ghana, interviews were conducted in Twi and Ga.

Ethics approval for the study was obtained from the ethics committee of CCDC (reference: IRB00006330) and the participating hospital (reference: IEC-486/07.10.2016, RP 5/2016, RP 34/2017), in India. In Ghana, ethical approval was obtained from the Ethical Review Committee of the Ghana Health Service (reference: GHS/RDD/ERC/Admin/App/16/189; GHS-ERC 06-092016). In the UK ethical approval was granted by the London School of Hygiene \& Tropical Medicine Research Ethics Committee (LSHTM Ethics Ref: 12046)

As the fundamental ethical principle for this study, we ensured the participants' anonymity throughout the data collection and analysis process. All participants were provided with an information sheet and consent form in their local language. Researchers at both sites read the information sheet and consent line by line to each participant and asked if they had any queries. Written consent was obtained from participants for recording interviews.

The data were collected between May and October 2017. The a priori themes that guided the interviews included disease perception, taking medicines, perception of the health systems, support network in the treatment, use and appropriation of technology. W asked participants questions such as: What has been your experience with cardiovascular disease?; Currently, are you taking medications for your disease?; and Do you take any medicines? How do you feel about that? Is it sometimes difficult to take them? Why? Do you feel these medicines help you? Or harm you?

\section{Analysis}

We explored data around six major themes, including disease perception/description of disease event, adherence to medication, and health systems barriers and facilitators. We usedCOM-B framework to analyse the data, which proposes that Capability, Opportunity and Motivation cause change in Behaviour (COM-B ${ }^{18}$ to address medication adherence. The context-specific emergent themes were added to expand the existing COM-B framework.

In contrast to previous unidimensional frameworks, COM-B is a comprehensive and solution-oriented framework that applies to various health-related behaviours. ${ }^{19}$ The COM-B framework helps in understanding specific behaviours and their causes. In this study, COM-B has been used to understand the health-related behaviours of patients in two different settings: India and Ghana.

The COM-B framework has three crucial components: capability, opportunity and motivation. The capability component has two sub-components: psychological and physical. Psychological capability deals with the cognitive ability of patients. Physical capability focuses on patients' ability to modify their lifestyle. The opportunity component of the framework also has two sub-components: physical and social. The physical opportunity comprises of cost of medicine, packaging, physical appearance, access to medicines and health services, regime complexity, communication between doctor and patients and the social support provided to patients. Social opportunity includes the stigma attached to the disease and the cultural beliefs that affect adherence. The motivation component revolves around reflective and automatic factors affecting a patients' motivation. The reflective factor includes patients' perception of illness, belief about the treatment and the outcome, whereas automatic motivation focuses on stimuli, mood or state of mind.

All the recorded interviews were transcribed and then translated into English by trained transcribers in India and Ghana. In India, the researcher who conducted the IDIs coded all the transcripts using QSR-NVivo 10. A team of two researchers coded transcripts in Ghana using QSR NVivo 11. A priori codes were derived from the COM-B framework, and the transcripts were coded using the interpretative approach, and deductive logic. This framework was adopted to guide the inquiry at the initial stage, and 
finally, a broader application of the themes and the concepts was developed. Thematic saturation was reached when no more themes were emerging from the data.

The evidence generated from the data was reconciled in the proposed framework. There were various linear stages followed in the analysis during and after the fieldwork. The researcher read and re-read the transcripts and field notes during and after the data collection to become familiar with the data. It also helped in identifying themes, following which labels were assigned to the data. Later, summaries of data under various labels were prepared, and initial themes were refined. Summary for each code and shared within the team, which included researchers from India, Ghana and the UK to identify overarching categories and later assigned themes and sub-themes. This process was discussed with the study investigators (including a senior qualitative researcher) for refinement on a regular basis. Following the COM-B framework ${ }^{18} 20$ existing themes were used to interpret data and the framework was expanded adding 10 themes under capability, opportunity and motivation domains. At the later stage, one more domain titled "overall adherence element and health system factors" was added, which specifically focused on context-specific reasons of non-adherence and health systems factors. This domain had two major themes on health care experience and trust in the facilities and reliance on alternative medication. Efforts were made to identify the association between themes until the "whole picture" emerged. Further patterns developed within the themes and concepts, and the original data were reflected constantly to ensure that participants' views and perceptions were accurately presented.

Researchers constantly referred to reflexivity notes and field diary at the time of data analysis in order to make sense of language, symbols, and metaphors used by the respondents. The researcher was also aware of her gender, age, affiliation while preparing the reflexivity notes. The researchers were also aware that the place of the interview might have shaped interview and interaction with the participant.

\section{Findings}

The analytical themes were derived from the COM-B framework (See Table 2).

\section{Capability}

The capability component helped in identifying the physical and psychological factors affecting the patients' adherence to medication.

\section{Psychological Capability Comprehension of Disease and Treatment}

In the Indian context, participants reported varying degree of understanding about their condition, diagnostic tests and treatment, and only a few participants had a comprehensive understanding of these.

"I do not have much expertise and information, but the disproportionate cholesterol ratio increases the tendency of clotting. I want a solution for this cholesterol proportion and clotting”. (IND: Male: Age 63)

In Ghana, some of the participants had not anticipated that they would develop CVD. Participants indicated that the onset of symptoms was often sudden and unexpected, resulting in hospitalisation.

"It was in January 2016 when I came to the hospital; I was feeling pain in my chest, so I came to the hospital ... I did not know what it was and he (doctor) told me that I had developed heart disease, so he referred me to a doctor ..." (GHN: Female: Age 67)

In both countries, female participants had limited information about their condition as in many instances they relied on other family members to communicate with their doctors.

"I did not know anything. I did not even know if I was given an injection. I remember being shifted from one room to another ... Later on, my children told me that the doctor had inserted something in my heart, but I could not get what it was." (IND: Female: Age 51)

\section{Cognitive Functioning}

This component helped in exploring patients' healthseeking behaviour. In Ghana, most participants shared that they adhered to the treatment plan to cure their condition.

"It is my duty to remind myself. My wife also reminds me, but it is my duty. I know that by 8 , I have to take the drug. Those that you will take the food before taking it is by me. When I finish, then I also take it but those that you take before taking the meal I take it." [GHN, Male, 53 years]

Some participants in India reported ignoring the primary symptoms of CVDs and delaying preventive treatment. They also discontinued their treatment after symptoms subsided.

"I stopped having the medicine from the current prescription for diabetes and medicines which were prescribed earlier. So I shared it with the doctor, and they continued 
Table 2 Major Analytical Themes and Sub-Themes: Expanding the *COM-B Framework

\begin{tabular}{|c|c|c|c|}
\hline Capability & Motivation & Opportunity & $\begin{array}{l}\text { Overall Adherence } \\
\text { Element and } \\
\text { Health System } \\
\text { Factors }\end{array}$ \\
\hline $\begin{array}{l}\text { The individual's physical } \\
\text { and psychological capacity } \\
\text { to engage in the behaviour } \\
\text { Psychological } \\
\text { Capacity to engage in the } \\
\text { necessary thought process } \\
\text { - Comprehension of dis- } \\
\text { ease and treatment } \\
\text { - Cognitive functioning (eg } \\
\text { memory, capacity for } \\
\text { judgement, thinking) } \\
\text { - Executive function (eg } \\
\text { capacity to plan) } \\
\text { - Medicine and Daily } \\
\text { Routine } \\
\text { - Medication Schedule } \\
\text { Physical } \\
\text { Capacity to engage in the } \\
\text { necessary physical } \\
\text { process } \\
\text { Physical capability to } \\
\text { adapt to lifestyle changes } \\
\text { (eg diet or social } \\
\text { behaviour) } \\
\text { Fexterity } \\
\text { Fatigue }\end{array}$ & $\begin{array}{l}\text { All brain process that energises and direct } \\
\text { behaviour } \\
\text { Reflective } \\
\text { Evaluations and plans } \\
\text { - Perception of illness (eg cause, chronic vs acute } \\
\text { etc.) } \\
\text { - Beliefs about treatment (eg necessity, efficacy, } \\
\text { concerns about current or future adverse } \\
\text { events, general aversion to taking medicines) } \\
\text { - Patients' Perception of Health } \\
\text { - Outcome expectancies } \\
\text { - Fear of Recurrence of Event } \\
\text { - Self-efficacy } \\
\text { Automatic } \\
\text { Emotions and impulses arising from associative } \\
\text { learning and/or innate dispositions } \\
\text { - Stimuli or cues for action } \\
\text { Mood state/disorder (eg depression and } \\
\text { anxiety) }\end{array}$ & $\begin{array}{l}\text { All factors lining outside the individual } \\
\text { that makes the performance of the } \\
\text { behaviour possible or prompt it } \\
\text { Physical } \\
\text { - Cost } \\
\text { - Access (eg availability of medication) } \\
\text { - Health Insurance } \\
\text { - Availability of medication } \\
\text { - Packaging } \\
\text { - Physical characteristics of medicine } \\
\text { (eg taste, smell, size, shape, route of } \\
\text { - administration) } \\
\text { - Regimen complexity } \\
\text { - Frequent Change in Medication } \\
\text { - Sack of Clarity in Prescription } \\
\text { - HCP-patient } \\
\text { - Lommunication } \\
\text { - Rimited Time for Consultation } \\
\text { - The } \\
\text { The cultural milieu that dictates the } \\
\text { way we think about things } \\
\text { disclosure }\end{array}$ & $\begin{array}{l}\text { These contextual } \\
\text { factors are specific to } \\
\text { LMICs } \\
\text { Adherence } \\
\text { Element } \\
\text { - Reasons for } \\
\text { adherence } \\
\text { - Reasons for non- } \\
\text { adherence } \\
\text { Health systems } \\
\text { element } \\
\text { - Health care experi- } \\
\text { ence and trust in } \\
\text { the facilities } \\
\text { - Reliance on alterna- } \\
\text { tive medication }\end{array}$ \\
\hline
\end{tabular}

Notes: Adapted from Jackson C, Barber N, Eliasson L, Weinman J. Applying COM-B to medication 980adherence: a suggested framework for research and intervention. EurJ Health Psychol. 2014;16:7-17. Copyright (c) 2016 Christina Jackson, Lina Eliasson, Nick Barber, John Weinman. This work is licensed under a Creative Commons Attribution 4.0 International License. ${ }^{18}$

the same (from the previous prescription) medication and also told me that the medication was suitable for my condition." (IND: Female: Age 41)

\section{Executive Function}

This refers to making and executing treatment plans. In both India and Ghana, some participants procured medicines and planned their visits to the doctor well in advance.

"I decided to take drugs at night since I do not want to fall asleep during the day while I might be in town or doing something. When I take it at night, then I can sleep. But if I forget to take it at night then I will not feel sleepy, so quickly I will go for it and take it before I can sleep." [GHN: Female: Age 37]

\section{Medicine and Daily Routine}

Taking the medication on time also became a part of the daily routine for a few participants. For some participants, family played a vital role and reminded participants for their medication.

"The hypertension medication I was told to take early in the morning, so when I am done brushing my teeth, I take the medication first thing in the morning.". (GHN: Female: Age 63)

\section{Medication Schedule}

Participants shared that it was easy for them to adhere to medication because of fewer prescribed medicines. It was easy for them to remember and take all medicines at once. 
"He (doctor) had advised me to have the medicine once in a day, so the night timing suits me well. I have food, and after that I have medicines." (IND: Male: Age 40)

\section{Physical Capability}

This refers to the individual's physical ability to adhere to their treatment.

\section{Unplanned Travel and Fatigue}

Some factors contributing to non-adherence were similar across age groups and gender. Unexpected travel and physical fatigue were frequently cited reasons for nonadherence.

"I forget medicine only if I have to travel, and I leave home during day time and reach my destination at night and cannot get back home that day itself. I miss medicine only if I am not able to return home." (IND: Male: Age 48)

\section{Dexterity}

In India, participants shared that they never sought help for medication, while in Ghana, participants reported waning ability to perform routine tasks, including medication. However, most participants had experienced a gradual improvement in their capacity to perform physical activities.

"First, I was able to wash clothes. I could go to the market and make a purchase, but I cannot do that right now. However, I have been told that it will get better. And when I get better, I will be able to do those things" (GHN: Female: Age 48)

\section{Opportunity}

It is crucial to explore the available opportunities for patients in LMICs, which make them adhere to medication, and how the lack of such opportunities hinders medication adherence.

\section{Physical Opportunity \\ Access}

Participants told that due to the distantly located hospital pharmacy, they face various difficulties in accessing medicines. In addition, the unavailability of medicines of a particular brand was a challenge for many participants.

"it is very difficult to find this medicine named Embeta R, which was prescribed by the doctor [name] here. I did not find it anywhere. I spent around INR 1000-1100 for conveyance to search this medicine." (IND: Male: Age 38)

\section{Cost of Medication}

In India, some of the participants reported facing challenges in affording medication. For participants from both India and Ghana, the cost of medical procedures and medicines resulted in non-adherence and discontinued treatment.

"When I went to the hospital once again and asked the doctors to examine me, they advised that another stent will be inserted in my artery. Listening to that, I started crying in front of my Aunt and told her that this is beyond my \{financial\} capacity". (IND: Male: Age 38)

\section{Health Insurance}

In Ghana, the National Health Insurance Scheme was helpful for participants, who could not afford expensive medication; however, the insurance did not cover all the drugs.

"No, you can see, I have the insurance booklet. However, when I go to the dispensary, I am told that the drug is not covered. So the doctor gave me a prescription to go and buy (medicines from the market) because I could not get them at the dispensary." (GHN: Female: Age 74)

\section{Availability of Medication}

In India, subsidised medicines, available at the hospital pharmacy, facilitated adherence to medication among participants. However, a few of them reported being unable to find some medicines at the hospital pharmacy prescribed by the doctor.

"Maybe because they are free and also generic medicines, so they are different in name since these generic medicines are less costly, that is why they are provided here (hospital pharmacy). This is what I think. This is the difference." (IND: Male: Age 42)"

In Ghana, there was a challenge with accessing drugs at the hospital pharmacy, where only a few medicines were covered under the National Health Insurance Scheme. Also, participants reported travelling from distant places and waiting for long hours to receive free medicines.

\footnotetext{
"When you come you will have to wait for a long time and spend all your time at the hospital" (GHN: Female: Age 66)
} 


\section{Physical Characteristics and Packaging of the Medicines}

Participants shared their experiences with medicines' shape and size. It was difficult for some participants in India to swallow the capsules, while in Ghana, no participant reported any such issues.

"The only issue is that when I take a capsule or the tabletlike Rozucor or Prolomet R $25 \mathrm{mg}$, they do not go down my throat easily and stay there for long, even if I drink a lot of water." (IND: Male: Age 68)

However, different packaging or different trade names of prescribed drugs were problematic for some participants. They refused to take medicines with different brand names.

"I will tell them I do not like it, if they say it's the same but different brand I do not like it, at times it might be fake so if you mix it with the drugs you are taking you might end up having some complications, or it will not work as expected, so I do not mix my drugs." (GHN: Female: Age 66)

\section{Regimen Complexity}

Majority of the participants did not find their, medication regimen complex, and it was not a barrier to medication adherence. They complied with prescribed medications.

"No, it's one pill daily, so that is why I take it only at night so I do not end up making it more than expected." [GHN: Female: Age 37]

\section{Frequent Change in Medication}

Frequent change in prescription adversely affected adherence among participants. Change in medication was observed at three levels: change in the brand name; change in regimen; and change in medication due to delayed recovery. Consequently, the participants resorted to alternative medicines for immediate results.

"I told him that sometimes I feel weak. I start gasping if I walk fast. This is what I told him, and he kept on changing the medicine accordingly, and I have come this far, and now I am taking two medicines daily". (IND: Male: Age 68)

\section{Healthcare Provider (HCP)-Patient Relationship/ Communication}

Many participants cited Communication with their doctor as a challenge. They generally preferred to receive advice from their doctors and health workers who were not always forthcoming.

"I think he (doctor) should be able to tell us what lead to the condition and also what not to do and also not to overthink. I think this will help us because I think the doctor knows more than I do" (GHN: Female: Age 60 years)

Usually, at the health facilities in countries like India and Ghana, it is difficult for doctors to communicate with every patient in detail due to the disproportionate doctorpatient ratio.

"They (doctor) do not give us any information. When you go, they will just ask you how you are doing, and normally you do not always see one doctor. Always there is a different doctor to attend to you and all that they do is to look in the folder history and rewrite the former medication for you. He will not explain anything to you, and all that he will do is to ask you to go and get the prescribed medication for you" (GHN: Female: Age 74 years)

\section{Lack of Clarity in Prescription}

In India and Ghana, participants mentioned having problems understanding their prescriptions, and they relied on the pharmacist to provide information on their medication. However, in many instances, people working at the pharmacy were not trained, pharmacists.

"We used to feel that this (OD, BD, BBF) is the name of the medicine. I used to think that OD means overdose. Yes, we can understand that zero (signifying number of doses) things, but we never got OD, HS, and BD etc. (IND: Male: Age 42)

"Here, pharmacists do not converse with patients, but you can ask them questions if you have any complaints. And even some of the people at the drug store and pharmacy are not pharmacists" [GHN: Female: Age 66]

\section{Limited Time for Consultation}

In India, the study was conducted at a tertiary care hospital, which provided specialised care to patients from all across the country. Due to low doctor-patient ratio, patients reported insufficient time for consultation with doctors.

"At this hospital, there were many patients. I went there once or twice if I had some problem. It used to be very suffocative during summer, and patients were huddled-up. Doctors did not have much time to give to the patients". (IND: Male: Age 63) 
Some participants got plenty of time with their doctor to discuss their health and related issues, while others faced challenges when doctors on duty were changed frequently. The other reason for the limited interaction between doctors and patients was a lower doctor to patient ratio.

"The doctor has no time. Yeah. They will ask you, what is your problem, any complain? No complaint then they see the card and give you the drug." [GHN: Male: Age, 59]

\section{Social Support}

Words of encouragement, prayers and financial support from friends, church and community members were valued by patients and helped them in medication adherence. Many participants shared that family members were very helpful in getting adequate treatment and in maintaining a healthy lifestyle.

"That was a difficult time for me. My children used to feed me on the bed or the table I was doing everything on my own, and suddenly, one day, I was not able to do anything. I even required help to go to the toilet in the beginning." (IND: Female: Age 56)

\section{Social Opportunity \\ Stigma of Disease}

Stigma attached to the disease demotivated many participants in both countries and had an impact on medication adherence. In some cases, participants did not disclose their medical condition to their family. Some participants did exhibit self-pity and self-stigmatisation tendencies because of their ill condition.

"Actually when I got married, I had many problems as I was so scared of my stroke that I used to develop palpitation when I was with my in-laws. If I had to travel and I develop palpitation, I used to make excuses to use the washroom, and I would look for a chemist shop to buy Disprin to be at ease." (IND: Female: Age 25)

We observed some context-specific expressions of stigma. In India, some young women seemed to describe hiding their condition from their in-laws, which affected the support received and ultimately, their treatment adherence. In contrast, in Ghana, some men expressed their worries in being found out at work.

\section{Religious and Cultural Belief}

In Ghana, some participants cited their religious belief and acts of spirituality, including prayers as a means to cure their disease. They also believed witchcraft to be the cause of their suffering.

"A brother of mine told me that this thing does not happen on people by heart, but maybe somebody has done it to you so when you go in the spirit they can tell you the cause of your sickness, and that's what I am expecting, I am very very serious, and it's true too" (GHN: Male: Age 73)

\section{Motivation}

Capability and opportunity have a direct impact on the motivation of a patient. Motivation is both reflective and automatic, which is shaped by personal experiences, perception and social milieu.

\section{Reflective}

\section{Perception of Illness}

Participants varied in their perception of illness. Some Indian participants shared that they developed heart disease due to the family history of the disease and mental stress.

"Basically, if I have no worries and no stress in my life, smaller stress are fine, but greater stress is when you have no clue what to do then in that circumstance I face the problem. I face a lot of challenge in that situation." (IND: Male: Age 63)

\section{Belief About Treatment}

Participants in India showed trust in their treatment, and they attributed their recovery to their medication and treatment.

"I am getting treatment from the expert doctor. Therefore, I have no worries about that. I do not have to think if the doctor is already thinking about me. I have medicines without any worry." (IND: Female: Age56)

Some of them also shared concerns about the side effects of medicines. In Ghana, participants even resorted to traditional and alternative medicines as they felt that the medicine prescribed by their doctor did not bring significant improvement in their condition.

"You know it is said that when you take the drug in plenty, it will trouble you later, oh no people tell me that a lot, even my sister's husband advice that I should take one because if I take them in plenty, it will give me side effect" (GHN: Female: Age 63) 
Participants also shared that various health conditions like diabetes, urinary problems and hypertension led to medication non-adherence, as they were unaware of the effect of the medicines for ASCVD on other health-related conditions that they had.

"Before then I have a urine-related problem that if I feel to urinate, then all of a sudden, the urine will come by itself and that exists still, and it still worries me." (GHN: Male: Age 73)

\section{Patients' Perception of Health}

Some participants shared that they discontinued medication upon feeling that their health had improved. Opinions and advice from non-medical staff, especially friends and family, also led to non-adherence in some cases.

"I started feeling well, and did not face BP issue for quite some time, so I thought that there is no need to carry on with medicines anymore. Therefore, I stopped taking medicines." (IND: Female: Age 51)

\section{Outcome Expectations}

In conditions like ASCVD, patients have to take medication for an extended period. Participants from Ghana felt that the medicines were not effective, and recovery was delayed.

"Sometimes I expect that the changes should be more than it is now looking at the number of drugs I take but am not God and like I have been told by the lady that it is a gradual process, sometimes I feel dizzy, and my arm hurts and my legs too." (GHN: Male: Age 63)

\section{Fear of Recurrence of Event}

In some other cases, physical pain and the fear of recurrence motivated participants to adhere to medication.

"I am afraid of facing the bad times again, and I do not want to face that, I underwent so much of pain and agony, because of that fear I can even stop eating food but I always keep medicines with me. Even today, I have the stock of medicines for coming six months." (IND: Female: Age 47)

\section{Self-Efficacy}

Most of the participants shared that they did not require any assistance in treatment and medication. In some cases, participants were altering the dose of medicines without consulting their doctor.
"No, the doctor had not told me to do so (alter the dose) I did that on my own. When I was going to [hospital], I had no other option, what would have I done. He had prescribed Atorva $20 \mathrm{mg}$ to me, and I had to take $40 \mathrm{mg}$ to compensate for a few missed doses." (IND: Male: Age 48)

\section{Automatic}

\section{Stimuli or Cues for Action}

The desire to live a long and a healthy life motivated many participants to adhere to medication, and they wanted to avoid the recurrence of an event that could be fatal.

"I had the information that skipping medication may lead to the recurrence of the attack. Therefore, I have not missed my medicine even for a day, and I always keep the extra stock of medicines. I am taking medicine in a disciplined manner". (IND: Female: Age 56)

Social support and family responsibility worked as an external stimulus for some participants to adhere to medication. Some of them followed the advice of family members and friends for their medication.

"The Financial problem is always there. But my priority is not to stop the medication due to any reason because I want to live, and there be no one to take care of my wife and children ..." (IND: Male: Age 40)

\section{Mood}

ASCVD affects the mental health and attitude of patients. Some participants reported developing mood swings and emotional instability, depression, fear of recurrence of stroke, and anger following the first event.

"Yes, there is one change that I have noticed, if there emerges any small or bigger issue at home or the workplace, I get anxious. And as I feel anxious I feel numbness in my head, and then I want complete isolation, and I do not want anyone to talk to me, neither about good thing nor a bad thing ..." (IND: Male: 48)

\section{Overall Adherence Element and Health System Factors Health Care Experience and Trust in the Facilities} In India, perception about the quality of care at the public sector hospital was quite positive among participants, as many of the participants had come to the government hospital after consulting doctors at various private hospitals. 
"I was satisfied that at least they (doctors) can diagnose my disease properly. They did not give any false hope and doing things properly. You feel good when you see that your doctors are connected to you, and they want to diagnose the disease adequately ...". (IND: Female: Age 25)

In Ghana, most participants seemed to be dissatisfied with the services they received at the public health facility, as the doctors were not attentive and did not share information related to diagnosis and treatment. They also complained about drug shortage at the hospital's pharmacy.

"For me, I have made up my mind to go to ridge hospital because the doctor who attends me is always going for inpatient wards rounds and she leaves the junior doctors around." (GHN: Female: Age 68)

\section{Reliance on Alternative Medication}

Some of the participants in Ghana shared that they relied on alternative and traditional medication, especially herbal medication. The few reasons given included the perceived lack of effectiveness of the allopathic medication and perceived effectiveness of herbal medicines and the side effects of the long-term treatment.

"My uncle is an herbalist, and he knows much about the herbs so after he saw the scan results, he told them that I had a stroke of the head. I was treated for five days with the herbal medicines and truly on the fourth day, I was able to move from one point to the other." (GHN: Female: Age 35)

In contrast, in India, some participants shared that Yoga and home remedies were beneficial for them, without the inconvenience of any side effects. However, these practices were complementary and did not have negative effect on participants' adherence to their medication.

"I had even visited Patanjali (Ayurveda clinic). There also, doctors told me that you could take the Ayurvedic medicine with the Allopathic medicines and there will not be any issue. These medicines are made of the barks and roots; thus, they will have no side effects. They instructed me to do Yoga." (IND: Female: Age 56)

\section{Discussion}

This qualitative study explored barriers and facilitators influencing medication adherence for ASCVD secondary prevention in two LMICs, India and Ghana. Adherence to medication, largely, was dependent on access, cost, physical and human characteristics of the health system, regimen complexity, and social support. We identified similarities in terms of barriers to medication in India and Ghana. The study manifests that the health system factors in LMICs played a vital role in limiting patient's physical opportunity to adhere to medication.

Due to lack of information in both India and Ghana, many participants ignored primary symptoms, which resulted in delayed treatment and inadvertent nonadherence. Previous studies have also established the importance of health literacy and adequate information about medication influences adherence. ${ }^{20,21}$

A distantly located health facility was an access barrier. Also, in both countries, limited communication between the doctor and patient was a major reason for discontent among patients. Many other studies have highlighted that patients did not adhere to medication because they lack adequate information about it. ${ }^{22,23}$ Though patients in India did not report major difficulties with their medical regimen, some were unaware of the codes used in the prescription to indicate the medication's timing. Thus, participants could not adhere to their prescription completely. In both countries, employees at the pharmacy explained medicines to the patients as medical language and clinical terms used in the prescription were beyond patients' comprehension, but they were not trained pharmacists. Medical vocabulary and clinical terms used in the prescription were beyond the comprehension of patients.

Financial constraints influencing adherence has been widely discussed in previous studies in LMICs. ${ }^{22-24}$ In this study, we found that some patients could not afford medication, which resulted in non-adherence. Lack of a well-stocked hospital pharmacy further aggravated problems for patients who could not afford costly medicines. Previous studies have also noted that irregular supply of better quality medicines at the pharmacy in the government hospitals acts as an access barrier to medication, resulting in poor medication adherence. ${ }^{25}$ Therefore, in LMICs, there is also a need for an adequate drug procurement system at the government hospitals to motivate patients to adhere to medication.

Unavailability of medicines with a particular brand name, as prescribed by the doctor, also resulted in nonadherence in India and Ghana. For many patients, unplanned travel, busy work schedules, and frequent changes in their prescription hindered medication compliance. Participants in both countries also shared their concerns about the side effects of long-term medication. 
Another study exploring the barriers to medication adherence among CVD patients noted that patients' perception of the side effects of long-term medication prevented them from adhering to medication. ${ }^{22}$

Though in previous studies, the complexity of regimen was found to be associated with non-adherence, ${ }^{26}$ we did not find this to be the case for our participants. In both countries, patients with clear cognitive functioning were able to take a decision for their treatment and complied with prescribed medication. Invariably, for male and female participant, health literacy played an important role and affected adherence. In the case of female participants, the male counterpart in the family took major decisions about their treatment. Most of the female participants were dependent on the male member of the family for medication and treatment. In both countries, participants who were self-dependent and took charge of their health and complied with medication. Other studies have also postulated that patients who can perform their daily activities independently and comfortably are mostly adherent to medication. ${ }^{23}$ Financial and emotional support from family members helped many participants in both countries adhere to medication. Many studies have underlined the family's positive role in improving adherence. ${ }^{22,27}$ Participants shared that their desire to live a healthy and long life made them adhere to medication. They also wanted to avoid recurrence of the cardiovascular event by complying with medication. Participants noted that medicines had become a part of their routine because they had been taking them for very long. Also, for a few participants, taking all the medicines together at a particular time was helpful in adhering to medication.

Though participants in India and Ghana shared various common factors, which hindered their medication adherence, there were some barriers, which were specific to each of the settings. In Ghana, the government provided health insurance to patients, which facilitates patients'medication. Though some of the participants were covered under government health insurance scheme as either they were working or had retired from the government services, many Indian participants were not covered by any government health insurance, which negatively impacted their medication adherence. However, since then government of India has launched Ayushman Bharat Pradhanmantri Jan Arogya Yojana in 2018, a flagship scheme programme to provide "health cover of Rs. 5 lakhs per family per year for secondary and tertiary care hospitalization to over 10.74 crores poor and vulnerable families". ${ }^{28}$
Additionally, in the Indian context, many participants could not share their medical condition with family because of social stigma attached to the disease, which influenced their medication negatively. Whereas in Ghana, reliance on alternative medication resulted in nonadherence among participants. There are various reasons for reliance on alternative medication, including easy availability, inexpensive medication, and trust in the efficacy of the alternative medication. The religious and cultural belief was very strong among participants in Ghana; in some cases, participants relied on prayers and did not adhere to medication after they were discharged from the hospital. The barriers to medication adherence identified in this study are broadly similar to the barriers in other settings. However, reliance on alternative medicines and lack of faith in modern medication was specific to Ghana. The participants, who could not understand their prescription, tend to miss medication or fail to adhere to their treatment, resulting in slow recovery or recurrence of the event. This had a significant impact on the patients' belief system, and they develop doubts about the treatment they were receiving.

This study has highlighted that the health system factors have dominantly influenced adherence to medication in India and Ghana. In both the countries, we found participants to be satisfied with their health care provided at the government hospitals, but limited time for consultation, lack of well-stocked pharmacy and unclear prescription negatively influenced adherence among participants. The study emphasises that the health system needs to be strengthened, and the patient's belief system needs to explore to address the issue of medication adherence in LMICs.

\section{Strengths and Limitations}

This study will help develop psychological understanding of patients' health-seeking behaviour and their complex interaction with the health systems component in LMICs. The previous frameworks ${ }^{18,29}$ have focused on human and health systems elements. In this study efforts have been made to explore contextual factors associated with health systems and patients' belief systems such as reliance on alternative medication, lack of clarity in prescription, and perception about public and private hospital. We came across these factors common in both India and Ghana while exploring barriers and facilitators using the COMB framework. These factors have customary/social, psychological and systemic roots in LMICs. 
While the identified barriers and facilitators were consistent in both countries, we also acknowledge that the study was confined to a single health facility in each country. We also recognise that the study was conducted at the tertiary referral hospitals and explored the barriers and facilitators beyond that could have helped develop a broader perspective. However, we made sure that we reached thematic saturation in both countries. Also, due to fewer female participants in the study, we could have missed specific barriers related to the challenges women face in accessing health care. The other limitation with this study -low number of subjects, however, we ensured that there is an adequate representation of men and women, different age groups and educational and socio-economic backgrounds and data saturation. There is a need to explore the barriers using a gender lens to make a holistic and robust theoretical background for medication adherence intervention.

Our study was conducted with a focus to generate a cumulative evidence base not only for policymaking but also to raise questions about local barriers and facilitators for further research. The study can inform health policymaking in LMICS where ASCVD is contributing to a huge number of deaths due to various socio-economic, psychological and systemic factors.

\section{Conclusion}

We have used the COM-B framework to explore contextual and health systems factors in LMICs influencing patients' adherence. Strict and comprehensive medication compliance was a challenge for both adherent and non-adherent participants across the gender and age group. The study highlights the importance of sharing patients' experience to design behaviour change interventions addressed to their specific problems to help them comply with their medication.

\section{Abbreviations}

CVD, cardiovascular disease; $\mathrm{NCD}$, non-communicable diseases; LMICs, low and middle income countries; ASCVD, atherosclerotic cardiovascular disease; COM-B, Capability Opportunity Motivation-Behaviour; HICs, high-income countries; IDIs, in-depth Interviews; FGD, Focused Group Discussion.

\section{Ethics Approval and Informed Consent}

Ethics approval for the study was obtained from the ethics committee of CCDC (reference: IRB00006330) and the participating hospital (reference: IEC-486/07.10.2016, RP 5/2016, RP 34/2017), in India. In Ghana, ethical approval was obtained from the Ethical Review Committee of the Ghana Health Service (reference: GHS/RDD/ERC/Admin/ App/16/189; GHS-ERC 06-09-2016). Written informed consent was obtained from the participants of the study. In the UK ethical approval was granted by the London School of Hygiene \& Tropical Medicine Research Ethics Committee (LSHTM Ethics Ref: 12046). We obtained written consent from all the participants to use and publish their anonymized responses in our research reports and manuscripts, and the study was conducted in accordance with the Declaration of Helsinki.

\section{Patient and Public Involvement}

Patients and/or the public were involved in the design, or conduct, or reporting, or dissemination plans of this research. Refer to the Methods section for further details.

\section{Author Contributions}

All authors made substantial contributions to conception and design, acquisition of data, or analysis and interpretation of data; took part in drafting the article or revising it critically for important intellectual content; agreed to submit to the current journal; gave final approval of the version to be published; and agree to be accountable for all aspects of the work.

\section{Funding}

The study is funded by UK'S Medical Research Council Grant MR/N021304/1 - via London School of Hygiene \& Tropical Medicine (LSHTM). The funding agency has no role in designing the study or in writing the manuscript.

\section{Disclosure}

The authors report no conflicts of interest in this work.

\section{References}

1. World Health Organization. Cardiovascular diseases (CVDs). Geneva: World Health Organization; 2017. Available from: https://www.who. int/en/news-room/fact-sheets/detail/cardiovascular-diseases-(cvds). Accessed August 27, 2019.

2. Gheorghe A, Griffith U, Murphy A, et al. The economic burden of cardiovascular disease and hypertension in low- and middle-income countries: a systematic review. BMC Public Health. 2018;18(1):975. doi:10.1186/s12889-018-5806-x

3. NCD Alliance. The global epidemic: non-communicable diseases (NCDs) are the world's \#1 killer, bringing hardship to rich and poor nations alike; 2015. Available from: https://ncdalliance.org/the-globalepidemic. Accessed August 28, 2019. 
4. Ruan Y, Guo Y, Zheng Y, et al. Cardiovascular disease (CVD) and associated risk factors among older adults in six low-and middle-income countries: results from SAGE wave 1. BMC Public Health. 2018;18(1):778. doi:10.1186/s12889-018-5653-9

5. Yusuf S, Hawken S, Ônpuu S, et al. Effect of potentially modifiable risk factors associated with myocardial infarction in 52 countries (the INTERHEART Study): Case-Control Study. Lancet. 2004;364 (9438):937-952. doi:10.1016/S0140-6736(04)17018-9

6. Kronish IM, Ye S. Adherence to cardiovascular medications: lessons learned and future directions. Prog Cardiovasc Dis. 2013;55 (6):590-600. doi:10.1016/j.pcad.2013.02.001

7. Ho PM, Bryson CL, Rumsfel JS. Medication adherence: its importance in cardiovascular outcomes. Circulation. 2009;119 (23):3028-3035. doi:10.1161/CIRCULATIONAHA.108.768986

8. Delamater AM. Improving patient adherence. Clin Diabetes. 2006;24 (2):71-77. doi:10.2337/diaclin.24.2.71

9. Perel P, Avezum A, Huffman M, et al. Reducing premature cardiovascular morbidity and mortality in people with atherosclerotic vascular disease. Glob Heart. 2015;10(2):99-110. doi:10.1016/j. gheart.2015.04.003

10. Mechael P, Batavia H, Kaonga N, et al. Barriers and Gaps Affecting Mhealth in Low and Middle Income Countries. Washington, DC: mHealth Alliance; 2010. Policy white paper.

11. World Health Organization. Risk of premature death from target NCDs data by country. Geneva: World Health Organization; 2018. Available from: http://apps.who.int/gho/data/view.main.2485. Accessed November 11, 2019.

12. Lamptey P, Laar A, Adler AJ, et al. Evaluation of a community-based hypertension improvement program (ComHIP) in Ghana: data from a baseline survey. BMC Public Health. 2017;17(1):368. doi:10.1186/ s12889-017-4260-5

13. Lloyd-Sherlock P, Beard J, Minicuci N, et al. Hypertension among older adults in low- and middle-income countries: prevalence, awareness and control. Int J Epidemiol. 2014;43(1):116-128. doi:10.1093/ ije/dyt 215

14. Prabhakaran D, Jeemon P, Roy A. Cardiovascular disease in India. Circulation. 2016;133(16):1605-1620. doi:10.1161/CIRCULATION AHA.114.008729

15. Subramanian SV, Corsi DJ, Subramanyam MA, et al. Jumping the gun: the problematic discourse on socioeconomic status and cardiovascular health in India. Int $J$ Epidemiol. 2013;42(5):1410-1426. doi:10.1093/ije/dyt017

16. Yusuf S. Two decades of progress in preventing vascular disease. Lancet. 2002;360(9326):2-3. doi:10.1016/S0140-6736(02)09358-3

17. Yusuf S, Islam S, Chow CK, et al. Use of secondary prevention drugs for cardiovascular disease in the community in high-income, middle-income, and low-income countries (the PURE Study): a prospective epidemiological survey. Lancet. 2011;378 (9798):1231-1243. doi:10.1016/S0140-6736(11)61215-4
18. Jackson C, Eliasson L, Barber N, Weinman J. Applying COM-B to medication adherence: a suggested framework for research and intervention. Eur J Health Psychol. 2014;16:7-17.

19. Michie S, van Stralen MM, West R. The behaviour change wheel: a new method for characterising and designing behaviour change interventions. Implement Sci. 2011;6(42). doi:10.1186/1748-59086-42

20. Lee SK, Kang BY, Kim HG, et al. Predictors of medication adherence in elderly patients with chronic diseases using support vector machine models. Healthc Inform Res. 2013;19(1):33-41. doi:10.4258/hir.2013.19.1.33

21. Saqlain M, Riaz A, Malik MN, et al. Medication adherence and its association with health literacy and performance in activities of daily livings among elderly hypertensive patients in Islamabad, Pakistan. Medicina (Kaunas). 2019;55(5):163. doi:10.3390/medicina55050163

22. Legido-Quigley H, Naheed A, Silva HA, et al. Patients' experiences on accessing health care services for management of hypertension in rural Bangladesh, Pakistan and Sri Lanka: a Qualitative Study. PLoS One. 2019;14(1):e0211100. doi:10.1371/journal.pone.0211100

23. Khatib R, Schwalm J-D, Yusuf S, et al. Patient and healthcare provider barriers to hypertension awareness, treatment and follow up: a systematic review and meta-analysis of qualitative and quantitative studies. PLoS One. 2014;9(1):e84238. doi:10.1371/journal. pone. 0084238

24. Maimaris W, Paty J, Perel P, et al. The influence of health systems on hypertension awareness, treatment, and control: a systematic literature review. PLoS Med. 2013;10(7):e1001490. doi:10.1371/journal. pmed.1001490

25. Balasubramanian A, Nair S, Rakesh PS, Leelamoni K. Adherence to treatment among hypertensives of rural Kerala, India. Fam Med Prim Care Rev. 2018;7(1):64-69. doi:10.4103/jfmpc.jfmpc_423_16

26. Donnan PT, MacDonald TM, Morris AD. Adherence to prescribed oral hypoglycaemic medication in a population of patients with type 2 diabetes: a Retrospective Cohort Study. Diabet Med. 2002;19 (4):279-284. doi:10.1046/j.1464-5491.2002.00689.x

27. Qureshi NN, Hatcher J, Chaturvedi N, et al. Effect of general practitioner education on adherence to antihypertensive drugs: cluster randomised controlled trial. BMJ. 2007;335(7628):1030. doi:10.1136/bmj.39360.617986.AE

28. Pradhan Mantri Jan Arogya Yojana. About Pradhan Mantri Jan Arogya Yojana (PM-JAY). New Delhi: National Health Authority, Government of India; 2018. Available from: https://pmjay.gov.in/ about/pmjay. Accessed October 5, 2020.

29. World Health Organisation. Adherence to long-term therapies. Evidence for action. Geneva: World Health Organization; 2003. Available from https:/www.who.int/chp/knowledge/publications/ adherence_full_report.pdf?ua=1. Accessed November 25, 2019.
Patient Preference and Adherence

\section{Publish your work in this journal}

Patient Preference and Adherence is an international, peer-reviewed, open access journal that focusing on the growing importance of patient preference and adherence throughout the therapeutic continuum. Patient satisfaction, acceptability, quality of life, compliance, persistence and their role in developing new therapeutic modalities and compounds to optimize clinical outcomes for existing disease states are major areas of interest for the journal. This journal has been accepted for indexing on PubMed Central. The manuscript management system is completely online and includes a very quick and fair peer-review system, which is all easy to use. Visit http:// www.dovepress.com/testimonials.php to read real quotes from published authors. 